\title{
ESTUDO COM ENFERMEIROS E MÉDICOS DA ATENÇÃO BÁSICA À SAÚDE: UMA ABORDAGEM SOCIOAMBIENTAL ${ }^{1}$ STUDY WITH PRIMARY CARE NURSES AND PHYSICIANS: A SOCIO-ENVIRONMENTAL APPROACH \\ ESTUDIO CON ENFERMEROS Y MÉDICOS DE LA ATENCIÓN PRIMARIA DE SALUD: UN ABORDAJE SOCIO-AMBIENTAL
}

\author{
Marta Regina Cezar-Važ2, Alisia Helena Weis ${ }^{3}$, Valdecir Zavarese da Costa 4 , Jorgana Fernanda de Souza Soares, \\ Clarice Alves Bonow', Letícia Silveira Cardoso', Cynthia Fontella Sant'Anna ${ }^{8}$, Maria Cristina Flores Soares?', \\ Ana Luiza Muccillo-Baisch ${ }^{10}$
}

${ }^{1}$ Trabalho financiado pelo Conselho Nacional de Desenvolvimento Científico e Tecnológico (CNPq) e Fundação de Amparo à Pesquisa do Rio Grande do Sul (FAPERGS). Desenvolvido no Laboratório de Estudos de Processos Socioambientais e Produção Coletiva de Saúde (LAMSA), da Fundação Universidade Federal do Rio Grande (FURG).

${ }^{2}$ Enfermeira. Doutora em Filosofia da Enfermagem. Professora Associado do Departamento de Enfermagem da FURG. Rio Grande do Sul, Brasil.

${ }^{3}$ Enfermeira. Mestre em Enfermagem. Ex-bolsista da Coordenação de Aperfeiçoamento de Pessoal de Nível Superior (CAPES). Rio Grande do Sul, Brasil.

${ }^{4}$ Mestrando do Programa de Pós-Graduação em Ciências da Saúde da FURG. Enfermeiro do Hospital Santa Casa do Rio Grande. Bolsista CAPES. Ex-bolsista de Iniciação Científica (IC) da FAPERGS. Rio Grande do Sul, Brasil.

${ }^{5}$ Enfermeira. Mestre em Enfermagem. Ex-Bolsista CAPES. Rio Grande do Sul, Brasil.

${ }^{6}$ Aluna do Curso de Graduação em Enfermagem da FURG. Bolsista IC/CNPq. Rio Grande do Sul, Brasil.

7 Aluna do Curso de Graduação em Enfermagem da FURG. Bolsista do Programa Institucional de Bolsas de Iniciação Científica (PIBIC/CNPq). Rio Grande do Sul, Brasil.

${ }^{8}$ Aluna do Curso de Graduação em Enfermagem da FURG. Bolsista IC/FAPERGS. Rio Grande do Sul, Brasil.

${ }^{9}$ Fisioterapeuta. Doutora em Fisiologia. Professora Adjunto do Departamento de Ciências Fisiológicas da FURG. Rio Grande do Sul, Brasil.

${ }^{10}$ Enfermeira. Doutora em Biologia da Saúde. Professora Associado do Departamento de Ciências Fisiológicas da FURG. Rio Grande do Sul, Brasil.

PALAVRAS-CHAVE: Atenção primária à saúde. Serviços de saúde. Saúde ambiental. Enfermagem em saúde pública.
RESUMO: Trata-se de estudo quantitativo sobre o processo organizativo do trabalho na Atenção Básica de Saúde, a partir de uma abordagem socioambiental. Os objetivos foram analisar como enfermeiras e médicos identificam as estratégias para trabalharem a relação entre saúde e ambiente; que problemas ambientais influenciam a saúde das comunidades e como eles atuam com as comunidades frente aos mesmos. Foram realizadas entrevistas estruturadas com 36 enfermeiros e 19 médicos, seguindo-se uma análise quantitativa e contextual. O quesito referente ao conhecimento das situações ambientais que implicam em riscos à saúde recebeu média significativamente maior dos enfermeiros $(p=0,000)$ e dos médicos $(p=0,042)$. A falta de saneamento básico, presença de vetores, manejo inadequado de resíduos sólidos e ruas sem pavimentação foram os problemas ambientais da comunidade que os entrevistados atribuíram as melhores médias $(\mathrm{p}=0,000)$. Conclui-se que a estrutura organizativa do trabalho na atenção básica à saúde, ainda não é influenciada significativamente pela relação saúde e ambiente.
KEYWORDS: Primary health care. Health services. Environmental health. Public health nursing.
ABSTRACT: This is a quantitative study which, using a socio-environmental approach, analyzed the organizational process of work in primary health care. The objectives were to analyze how nurses and physicians identified strategies in order to work the relationship between health and the environment; which environmental problems influence the health oc communities; and how they act as communities in the face of such problems. Structured interviews were carried out with 36 nurses and 19 physicians in two cities in southern Brazil, followed with quantitative and contextual analysis. The question referring to knowledge of the environmental situations which imply effective or potential health risks received a significantly higher average from the nurses $(p=0,000)$ and from the physicians $(p=0,042)$. The lack of basic sanitation, the presence of vectors, inadequate handling of solid residues, and non-paved streets were the environmental problems of the community to which these workers attributed the best averages $(p=0,000)$. It can thus be concluded that the organizational structure of work in primary health care still is not significantly influenced by the health and environment relationship.
Marta Regina Cezar-Vaz

Endereço: Av. Presidente Vargas, 323, casa 13. Parque.

96.202-100 - Parque, Rio Grande, RS.

E-mail: cezarvaz@vetorial.net
Artigo original: Pesquisa

Recebido em: 16 de abril de 2007 Aprovação final: 08 de outubro de 2007 
PALAVRAS ClAVE: Atención primariadesalud. Servicios de salud. Salud ambiental. Enfermería en salud pública.
RESUMEN: Se trata de un estudio cuantitativo, con abordaje socio-ambiental, en el cual se analizó la organización del trabajo en la atención básica a la salud. Los objetivos fueron: analizar cómo las enfermeras y los médicos identifican las estrategias para trabajar la relación salud y el ambiente; qué problemas ambientales influencian la salud de las comunidades, y cómo ellos actúan con las comunidades ante los mismos. Para el estudio se realizaron entrevistas estructuradas a 36 enfermeros y 19 médicos, además de un análisis cuantitativo y contextual. El conocimiento de las situaciones ambientales que implican en riesgos efectivos o potenciales a la salud, recibió media significativamente mayor de los enfermeros $(\mathrm{p}=0,000)$ y de los médicos $(\mathrm{p}=0,042)$. La falta de saneamiento básico, la presencia de vectores, el manejo inadecuado de los residuos sólidos y las calles sin pavimentar fueran los problemas ambientales de la comunidad que los entrevistados atribuyeron las mejores medias $(p=0,000)$. Se concluye que la estructura organizativa del trabajo en la atención primaria de salud, aún no es influenciada significativamente por la relación salud y ambiente.

\section{INTRODUÇÃO}

O presente texto compõe o produto de uma investigação do processo de trabalho em Saúde Pública, na especificidade do trabalho desenvolvido na rede de Atenção Básica à Saúde de dois municípios vizinhos no extremo sul do Rio Grande do Sul; o município do Rio Grande e o município de São José do Norte, ambos localizados às margens do estuário da Lagoa dos Patos e pertencentes a $3{ }^{\text {a }}$ Coordenadoria Regional de Saúde. O foco da investigação foi o cotidiano das práticas constituintes deste trabalho e o conjunto de saberes que o suporta, tendo por finalidade apreender a aproximação do processo organizativo do trabalho à abordagem socioambiental preconizada pela Organização Mundial de Saúde (OMS). ${ }^{1}$

A partir do que foi definido como a estratégia da Atenção Primária Ambiental (APA), ${ }^{1}$ esta abordagem possibilitaria a ampliação da compreensão da relação entre saúde e ambiente, na direção da qualidade de vida dos indivíduos e da sustentabilidade dos espaços comunitários; ${ }^{2-3}$ sustentabilidade como autogestão, ou seja, como "[...] a participação direta na apropriação e transformação de seu ambiente para alcançar um desenvolvimento igualitário, sustentável e duradouro".2:368

Para melhor explicitar no texto, a compreensão do conteúdo constituinte deste enfoque socioambiental, ${ }^{2-3}$ apresenta-se na sequiência, o conjunto de características-componentes necessárias à estratégia da APA: a ação construtiva e integrativa (estratégias) da relação entre saúde e ambiente; a compreensão acerca dos problemas ambientais locais e a influência destes na saúde das comunidades; e as estratégias educativas com a comunidade e outros setores públicos que fortaleçam a gerência integrativa e participativa, por meio da identificação de problemas, da co-responsabilização na preservação e na promoção de ambientes por parte da comunidade e dos órgãos públicos.

Esta compreensão ampliada da atenção primária abrangendo a extensão ambiental do processo de saúde dos indivíduos e comunidades, implica então, na promoção da saúde ambiental localmente, procurando o desenvolvimento de estratégias de participação social, a partir do conhecimento, identificação e solução dos problemas ambientais que influenciam a saúde e limitam a qualidade de vida e a sustentabilidade. ${ }^{1}$

É uma forma de focar a saúde, embasada em práticas de ajuda mútua e relações solidárias no enfrentamento dos problemas, ${ }^{4}$ na qual a expressão atenção primária adquire o significado de uma estratégia de ação ambiental, calcada na prevenção e participação em nível local, a qual reconhece o direito dos seres humanos de viver em um ambiente saudável e o de ser informado sobreainfluência dos problemas ambientais na sua saúde, bem-estar e sobrevivência, posicionandoos como co-responsáveis na proteção, conservação e recuperação ambiental e da saúde. ${ }^{1}$

Neste enfoque, o processo de trabalho na atenção em saúde se potencializa na direção de ações interativas e participativas, possíveis a partir da ampliação da decisão, da programação, do planejamento, da supervisão e coordenação do processo de trabalho para a consecução estratégica da saúde na dimensão socioambiental. Comaestrutura redimensionada, pode-se, então, alterar o saber gerencial coletivo. ${ }^{5}$

Naestrutura ampliada, a interdisciplinaridade ${ }^{5}$ se constitui em característica a priori do enfoque socioambiental..$^{2 \cdot 3}$ A proposição desta característica transparece no conceito de saúde ${ }^{6}$ e na sua constituição ambiental, na qual identifica-se a positividade do conjunto de ações no trabalho estar na dimensão da cooperação, ${ }^{7}$ incluindo ações que projetam o trabalho e aquelas que movimentam e executam o próprio trabalho, ou seja, formas democráticas para desenvolvê-lo - produzindo cooperação.? Por meio das ações cooperativas de saúde, em realidades concretas, centradas na qualidade de vida dos seres humanos e no seu ambiente, ${ }^{4}$ se potencializa a interação dos trabalhadores de saúde com os indivíduos, as famílias e as comunidades. 
Neste contexto interdisciplinar é possível pensar que, ao assumir no processo de trabalho a possibilidade dedesenvolvereconsolidaraçõescooperativas, também se assume a possibilidade do enfoque socioambiental animar a produção do conhecimento interdisciplinar na área da saúde e da enfermagem, em particular, no campo da saúde coletiva socioambiental.

Salienta-se que essa aproximação à abordagem socioambiental em saúde no estudo, tendo como cenário o trabalho da rede básica de saúde, foi proposta pela inexistência da constituição formal de Centros de APA, ${ }^{1}$ até o momento da pesquisa, na estrutura de gestão pública dos municípios.

Com o pressuposto de que as políticas públicas para a saúde, ainda que seguindo princípios formais, adquirem expressão por meiode diretrizes operacionais construídas no próprio cotidiano das práticas, as quais passam a nortear o trabalho no nível básico da assistência, se entendeu ser possível identificar características dessa estratégia socioambiental já incluídas no trabalho operado por esses profissionais.

Seguindo a proposta de investigar a aproximação do processo organizativo do trabalho na atenção básica à saúde à abordagem socioambiental, ${ }^{2-3}$ inserida na estratégia da APA, preconizada pela OMS, este estudo teve como objetivos específicos apreender como os trabalhadores atuantes na atenção básica à saúde, especificamente os enfermeiros e os médicos, percebem as estratégias para trabalharem com a relação entre saúde e ambiente, que problemas ambientais identificam como influentes na saúde da comunidade e como atuam profissionalmente com as comunidades frente aos problemas ambientais identificados.

\section{METODOLOGIA}

O estudo tem a forma descritiva, abrangendo um corte transversal com análise quantitativa dos dados, do processo de trabalho em saúde desenvolvido na rede básica dos serviços públicos de saúde, incluindo o conjunto de Unidades com equipes de Saúde da Família (USF) e Unidades Básicas de Saúde (UBS), dos municípios do Rio Grande e São José do Norte, integrantes da $3^{\text {a }}$ Coordenadoria Regional de Saúde do estado do Rio Grande do Sul ( $\left.3{ }^{\text {a }} \mathrm{CRS} / \mathrm{RS}\right)$. Esse conjunto de unidades, representa a porta de entrada da demanda em saúde, no atendimento do Sistema Único de Saúde (SUS) na região pesquisada.

Para a seleção dos participantes do estudo foram considerados os critérios de tempo de atuação na rede básica, não inferior a um ano, e a disposição em parti- ciparda pesquisa. A amostra acidental ficou constituída por 55 trabalhadores (36 enfermeiros e 19 médicos), representando aproximadamente $25 \%$ da população dostrabalhadores. A maior partedostrabalhadoresatua no município do Rio Grande, que possui um número maior de unidades de saúde.

Os participantes foram informados quanto ao objetivo do estudo e ao procedimento da entrevista. Em consonância com as diretrizes e normas que regulam as pesquisas envolvendo seres humanos estabelecidas pela Resolução No 196/96 do Conselho Nacional de Saúde, todos os entrevistados expressaram por escrito o consentimento pós-informado, para os quais foi assegurado o direito de desistência da participação sem qualquer ônus e preservado o anonimato por meio da não identificação pessoal, dos locais de trabalho e dos municípios. A proposta de pesquisa foi previamente aprovada pelo Comitê de Ética em Pesquisa na Área da Saúde, da Pró-Reitoria de Pesquisa e Pós-Graduação da FURG, sob o processo $\mathrm{N}^{\circ}$ 23116.002761/2005-17, e parecer $\mathrm{N}^{\circ}$ 036/2006. Também, foi solicitada previamente a concordância para realização da pesquisa junto à $3{ }^{\mathrm{a}} \mathrm{CRS} / \mathrm{RS}$.

As entrevistas individuais seguiram um questionário estruturado e pré-codificado, composto por 51 perguntas fechadas de múltipla escolha. Para as respostas foi utilizada uma escala de grau de importância, contendo notas de 0 a 10. Em cada questão o entrevistado foi solicitado a dar a nota ao quesito perguntado, considerando a sua realidade de trabalho particular e coletiva.

O instrumento foi averiguado por meio do teste piloto aplicado a sete enfermeiros que exerciam diferentes funções dentro dos serviços públicos de saúde. Além disso, a confiabilidade do instrumento foi testada através do cálculo do "alpha de Cronbach" que mede a fidedignidade do questionário $(>0,70)$. O coeficiente de fidedignidade do instrumento de pesquisa foi de 0,99 .

O banco de dados foi constituído a partir do software Epi Info $6.04 \mathrm{~d}$. Os dados foram analisados quanto ao cálculo da média, desvio padrão e análise de variância seguida do teste post hoc - Honestly Significant Difference (HSD) e foram considerados significantes os valores onde $\mathrm{p}<0,05$.

Para a análise dos elementos básicos importantes da relação saúde e ambiente no trabalho, as variáveis foram agrupadas e analisadas como a seguir.

Grupo 1 - estratégias participativas no trabalho da atenção básica à saúde para a ação construtiva e 
integrativa da relação entre saúde e ambiente, incluindo: - participação na definição das políticas públicas relacionadas ao meio ambiente e a saúde; - participação na construção de conhecimento acerca das situações ambientais do município que impliquem em riscos efetivos ou potenciais à saúde humana; - participação em projetos de intervenção ambiental para controlar os riscos ambientais na prevenção e promoção da saúde coletiva e individual; - participação na avaliação dos impactos causados por produtos químicos, agrotóxicos e outros produtos tóxicos na saúde da população; - participação na construção de indicadores de vigilância.

Grupo 2 - compreensão dos trabalhadores acerca dos problemas ambientais locais e a influência destes na saúde da comunidade: - falta de saneamento básico (sistema de esgoto); - contaminação atmosférica (industrial, doméstica, automotivas) que atinge a comunidade; - contaminação da água que é utilizada pela população de abrangência do trabalho; - uso indevido dos solos; - vetores de doenças; - ruas sem pavimentação; - falta de áreas verdes; - alimentos contaminados por agrotóxicos ou outras substâncias; - manejo inadequado de resíduos sólidos e domésticos (lixo, por exemplo); - erosão e desmatamento; - utilização de agrotóxicos em plantações.

Grupo 3 - estratégias de ação da gerência integrativa e participativa com a comunidade local e com outros setores públicos: - atividades de educação em saúde que viabilizem a identificação de problemas pela população de forma que cada indivíduo reconheça sua co-responsabilidade na preservação de ambientes, bem como promover o conhecimento das responsabilidades dos órgãos públicos; - atividades integrativas com os serviços que atuam diretamente com os diferentes problemas ambientais identificados (vigilância à saúde, secretaria do meio ambiente, serviços urbanos entre outros, ligados aos órgãos públicos).

\section{RESULTADOS}

A amostra foi constituída por 36 enfermeiros e 19 médicos, sendo 29 trabalhadores (17 enfermeiros e 12 médicos), vinculados as USF, e 26 (19 enfermeiros e 7 médicos) vinculados as UBS. Otempo médio de atuação nos respectivos serviços ficou em 2 anos e 5 meses. A idade dos trabalhadores variou entre 25 e 63 anos, encontrando-se a média de $39 \pm 9$ anos. Quanto ao sexo, houve o predomínio de mulheres (46 trabalhadoras).

A Tabela 1 resume os valores das variáveis reunidas no Grupo 1. O segmento dos enfermeiros tanto das UBS como das USF, valorizou o quesito referente ao conhecimento das situações ambientais do município que impliquem em riscos efetivos ou potenciais à saúde humanacom médiasignificantemente maior $(\mathrm{p}=0,000)$ em relação aos demais quesitos. Na análise comparativa não foi encontrada diferença significativa $(\mathrm{p}=0,289)$ entre as avaliações dos enfermeiros dessas unidades em relação as variáveis reunidas no Grupo 1.

Tabela 1 - Estratégias utilizadas por enfermeiros e médicos das UBS e USF para trabalhar com a relação entre saúde e ambiente. Rio Grande do Sul, 2006.

\begin{tabular}{|c|c|c|c|c|c|c|}
\hline \multirow[t]{2}{*}{$\begin{array}{l}\text { Estratégias utilizadas para trabalhar } \\
\text { com a relação entre saúde e ambiente }\end{array}$} & \multicolumn{2}{|c|}{$\begin{array}{l}\text { UBS } \\
\text { Categoria(n) }\end{array}$} & \multicolumn{2}{|c|}{$\begin{array}{c}\text { USF } \\
\text { Categoria(n) }\end{array}$} & \multicolumn{2}{|c|}{$\begin{array}{l}\text { USB+USF } \\
\text { Categoria(n) }\end{array}$} \\
\hline & $\begin{array}{l}\text { Enfermeiros } \\
\text { (19) }\end{array}$ & $\begin{array}{l}\text { Médicos } \\
(07)\end{array}$ & $\begin{array}{l}\text { Enfermeiros } \\
(17)\end{array}$ & $\begin{array}{l}\text { Médicos } \\
(12)\end{array}$ & $\begin{array}{l}\text { Enfermeiros } \\
\qquad(36)\end{array}$ & $\begin{array}{l}\text { Médicos } \\
\text { (19) }\end{array}$ \\
\hline $\begin{array}{l}\text { Participação nas políticas públicas } \\
\text { relacionadas ao meio ambiente e a Saúde }\end{array}$ & $5,26 \pm 2,86$ & $5,00 \pm 4,20$ & $6,00 \pm 3,14$ & $4,42 \pm 3,63$ & $5,61 \pm 2,98^{b}$ & $4,53 \pm 3,62^{b}$ \\
\hline $\begin{array}{l}\text { Conhecimento das situações ambientais } \\
\text { que impliquem em riscos efetivos ou } \\
\text { potenciais à saúde humana }\end{array}$ & $7,32 \pm 2,54$ & $8,17 \pm 2,14$ & $7,29 \pm 2,44$ & $5,58 \pm 3,00$ & $7,30 \pm 2,46^{a}$ & $6,374 \pm 2,89^{a}$ \\
\hline $\begin{array}{l}\text { Participação em projetos de intervenção } \\
\text { ambiental para controlar os riscos } \\
\text { ambientais na prevenção e promoção da } \\
\text { saúde coletiva e individual }\end{array}$ & $4,37 \pm 3,29$ & $6,17 \pm 3,71$ & $6,35 \pm 2,69$ & $4,83 \pm 3,49$ & $5,30 \pm 3,14^{b}$ & $5,0 \pm 3,62^{b}$ \\
\hline $\begin{array}{l}\text { Avaliação dos impactos causados produtos } \\
\text { tóxicos na saúde da população }\end{array}$ & $4,84 \pm 3,61$ & $5,83 \pm 3,43$ & $5,65 \pm 2,62$ & $4,67 \pm 3,63$ & $5,22 \pm 3,16^{b}$ & $4,84 \pm 3,53^{b}$ \\
\hline Construção de indicadores de vigilância & $5,00 \pm 3,25$ & $6,33 \pm 3,98$ & $6,53 \pm 2,85$ & $3,83 \pm 2,98$ & $5,72 \pm 3,12^{b}$ & $4,54 \pm 3,45^{b}$ \\
\hline
\end{tabular}

Os valores representam a média \pm desvio padrão.

Letras diferentes mostram diferença significativa $(p<0,05)$ entre as médias totais dos itens em uma mesma categoria de trabalhadores (enfermeiros ou médicos). 
$\mathrm{Na}$ análise específica para o grupo dos médicos das USF e UBS, foi verificado que os mesmos atribuíram nota média significantemente maior $(\mathrm{p}=0,042)$ ao item conhecimento das situações ambientais do município que impliquem em riscos efetivos ou potenciais à saúde humana. Na comparação entre os dois grupos de médicos, não foi constatada diferença significativa $(\mathrm{p}=0,610)$ entre as avaliações dos médicos dessas unidades (Tabela 1).

Considerando o total de 55 trabalhadores, não foi encontrada diferença significativa $(p=0,244)$ entre as médias dos quesitos referentes às estratégias utilizadas na gestão participativa para trabalhar na relação saúde ambiente, na comparação com o local de atuação, UBS ou USF.
Quanto aos problemas ambientais identificados pelo conjunto dos enfermeiros de UBS e USF como exercendo influência sobre a saúde da comunidade (Grupo 2), os quesitos como a falta de saneamento básico, presença de vetores de doenças, manejo inadequado de resíduos sólidos e domésticos e ruas sem $\mathrm{pa-}$ vimentaçãoforam significantemente $(p=0,000)$ melhor avaliados que os quesitos utilização de agrotóxicos em plantações, alimentoscontaminados poragrotóxicos ou outras substâncias, uso indevido dos solos e erosão e desmatamento. Quando comparados os dois grupos de enfermeiros, não foi constatada diferença significativa $(p=0,407)$ entre as médias das respostas dos trabalhadores das UBS e das USF (Tabela 2).

Tabela 2 - Problemas ambientais que influenciam a saúde da comunidade, identificados por enfermeiros e médicos das UBS e USF. Rio Grande do Sul, 2006.

\begin{tabular}{|c|c|c|c|c|c|c|}
\hline \multirow[t]{2}{*}{$\begin{array}{l}\text { Problemas ambientais que influenciam } \\
\text { a saúde da comunidade }\end{array}$} & \multicolumn{2}{|c|}{$\begin{array}{c}\text { UBS } \\
\text { Categoria(n) }\end{array}$} & \multicolumn{2}{|c|}{$\begin{array}{c}\text { USF } \\
\text { Categoria(n) }\end{array}$} & \multicolumn{2}{|c|}{$\begin{array}{l}\text { USB+USF } \\
\text { Categoria(n) }\end{array}$} \\
\hline & $\begin{array}{l}\text { Enfermeiros } \\
\quad(19)\end{array}$ & $\begin{array}{l}\text { Médicos } \\
(07)\end{array}$ & $\begin{array}{l}\text { Enfermeiros } \\
\quad(17)\end{array}$ & $\begin{array}{l}\text { Médicos } \\
\quad(12)\end{array}$ & $\begin{array}{l}\text { Enfermeiros } \\
\quad(36)\end{array}$ & $\begin{array}{l}\text { Médicos } \\
\text { (19) }\end{array}$ \\
\hline Saneamento básico (sistema de esgoto) & $7,89 \pm 2,71$ & $9,50 \pm 0,84$ & $7,88 \pm 3,3$ & $8,92 \pm 1,88$ & $8,11 \pm 2,69^{a}$ & $8,89 \pm 1,82^{a}$ \\
\hline $\begin{array}{l}\text { Contaminação atmosférica (industrial, } \\
\text { doméstica, automotivas) }\end{array}$ & $7,42 \pm 2,27$ & $6,83 \pm 3,43$ & $7,06 \pm 3,17$ & $7,17 \pm 3,01$ & $7,17 \pm 2,69^{a b}$ & $6,73 \pm 3,28^{\text {cdef }}$ \\
\hline Contaminação da água & $6,05 \pm 3,27$ & $7,83 \pm 2,04$ & $8,19 \pm 1,91$ & $7,42 \pm 3,63$ & $7,02 \pm 2,90^{\mathrm{ab}}$ & $7,42 \pm 3,09^{\text {bcde }}$ \\
\hline Uso indevido dos solos & $5,10 \pm 3,35$ & $5,17 \pm 3,54$ & $7,24 \pm 2,70$ & $7,00 \pm 3,69$ & $6,11 \pm 3,25^{\mathrm{bc}}$ & $6,05 \pm 3,83^{\text {efg }}$ \\
\hline Presença de vetores de doenças & $7,42 \pm 2,65$ & $8,50 \pm 2,51$ & $8,35 \pm 1,87$ & $8,67 \pm 2,31$ & $7,94 \pm 2,31^{a}$ & $8,21 \pm 2,83^{a b}$ \\
\hline Ruas sem pavimentação & $7,79 \pm 2,49$ & $7,67 \pm 4,08$ & $7,35 \pm 3,60$ & $8,42 \pm 3,50$ & $7,80 \pm 2,77^{a}$ & $7,74 \pm 3,96^{\mathrm{abcd}}$ \\
\hline Falta de áreas verdes & $6,74 \pm 2,54$ & $6,33 \pm 3,93$ & $7,00 \pm 2,98$ & $7,08 \pm 3,50$ & $7,05 \pm 2,48^{\mathrm{ab}}$ & $6,47 \pm 3,79^{\text {defg }}$ \\
\hline $\begin{array}{l}\text { Alimentos contaminados por agrotóxicos } \\
\text { ou outras substâncias }\end{array}$ & $6,00 \pm 3,45$ & $5,83 \pm 3,76$ & $6,29 \pm 3,12$ & $6,33 \pm 3,39$ & $6,31 \pm 3,12^{\mathrm{bc}}$ & $5,84 \pm 3,60^{f g}$ \\
\hline $\begin{array}{l}\text { Manejo inadequado de resíduos sólidos e } \\
\text { domésticos (lixo, etc.) }\end{array}$ & $7,58 \pm 3,08$ & $9,33 \pm 1,21$ & $7,71 \pm 3,12$ & $8,00 \pm 3,22$ & $7,86 \pm 2,79^{\mathrm{a}}$ & $8,0 \pm 3,29 a b c$ \\
\hline Erosão e desmatamento & $5,63 \pm 3,64$ & $3,83 \pm 3,66$ & $5,59 \pm 3,57$ & $6,17 \pm 3,24$ & $5,77 \pm 3,47^{c}$ & $5,10 \pm 3,58^{g}$ \\
\hline Utilização de agrotóxicos em plantações & $5,95 \pm 4,16$ & $4,50 \pm 4,23$ & $6,47 \pm 2,85$ & $6,58 \pm 3,03$ & $6,37 \pm 3,44^{\mathrm{bc}}$ & $5,57 \pm 3,65^{f g}$ \\
\hline
\end{tabular}

Os valores representam a média \pm desvio padrão.

Letras diferentes mostram diferença significativa $(\mathrm{p}<0,05)$ entre as médias totais dos itens em uma mesma categoria de trabalhadores (enfermeiros e médicos).

No que se refere aos problemas ambientais identificados pelo conjunto dos médicos das UBS e USF, como exercendo influência sobre a saúde da comunidade, o item saneamento básico foi o que recebeu média significantemente maior $(p=0,000)$ que os demais, exceto com relação aos quesitos presença de vetores de doença, ruas sem pavimentação e manejo inadequado de resíduos sólidos e domésticos (Tabela 2).
A análise ajustada para o local de trabalho (UBS ou USF), não mostrou diferença significativa $(p=0,444)$ entre os valores atribuídos pelos médicos dessas unidades na referência dos problemas ambientais que influenciam a saúde da comunidade.

Considerando a amostra de 55 trabalhadores, não foi encontrada diferença significativa $(p=0,427)$ entre as médias dos quesitos na comparação com o local de atuação, tanto em UBS como em USF. 
Tabela 3 - Forma de atuação dos trabalhadores das UBS e USF com as comunidades frente aos problemas ambientais identificados. Rio Grande do Sul, 2006.

\begin{tabular}{|c|c|c|c|c|c|c|}
\hline \multirow[t]{2}{*}{$\begin{array}{l}\text { Atuação profissional com as } \\
\text { comunidades frente aos problemas } \\
\text { ambientais identificados }\end{array}$} & \multicolumn{2}{|c|}{$\begin{array}{c}\text { UBS } \\
\text { Categoria(n) }\end{array}$} & \multicolumn{2}{|c|}{$\begin{array}{c}\text { USF } \\
\text { Categoria(n) }\end{array}$} & \multicolumn{2}{|c|}{$\begin{array}{l}\text { USB+USF } \\
\text { Categoria(n) }\end{array}$} \\
\hline & $\begin{array}{c}\text { Enfermeiros } \\
\text { (19) }\end{array}$ & $\begin{array}{c}\text { Médicos } \\
\text { (07) }\end{array}$ & $\begin{array}{c}\text { Enfermeiros } \\
\quad(17)\end{array}$ & $\begin{array}{l}\text { Médicos } \\
\text { (12) }\end{array}$ & $\begin{array}{c}\text { Enfermeiros } \\
(36)\end{array}$ & $\begin{array}{l}\text { Médicos } \\
\text { (19) }\end{array}$ \\
\hline $\begin{array}{l}\text { Atividades de educação em saúde que } \\
\text { viabilizem a identificação de problemas pel } \\
\text { população de forma que cada indivíduo } \\
\text { reconheça sua co-responsabilidade } \\
\text { na preservação de ambientes, bem } \\
\text { como promover o conhecimento das } \\
\text { responsabilidades dos órgãos públicos }\end{array}$ & $6,26 \pm 3,00$ & $6,83 \pm 3,76$ & $8,00 \pm 2,18$ & $7,17 \pm 2,66$ & $7,08 \pm 2,75^{a}$ & $6,74 \pm 3,19^{a}$ \\
\hline $\begin{array}{l}\text { Atividades integrativas com os serviços } \\
\text { que atuam diretamente com os diferentes } \\
\text { problemas ambientais identificados } \\
\text { (vigilância à saúde, secretaria do meio } \\
\text { ambiente, serviços urbanos entre outros, } \\
\text { ligados aos órgãos públicos) }\end{array}$ & $6,11 \pm 3,25$ & $6,50 \pm 3,02$ & $7,18 \pm 2,40$ & $5,00 \pm 2,98$ & $6,61 \pm 2,89^{a}$ & $5,26 \pm 3,08^{b}$ \\
\hline
\end{tabular}

Os valores representam a média \pm desvio padrão.

Letras diferentes mostram diferença significativa $(\mathrm{p}<0,05)$ entre as médias totais dos itens em uma mesma categoria de trabalhadores (enfermeiros ou médicos).

Com relação as variáveis do Grupo 3, que identificavam a forma de atuação junto às comunidades frente aos problemas ambientais, o conjunto dos enfermeiros das UBS e USF, como se observa na Tabela 3, não avaliou de forma significativamente diferente $(p=0,369)$ os itens analisados. Também não foi observada diferença significativa entre as avaliações em separado dos dois grupos de enfermeiros (UBS + USF).

No tocante aos médicos de UBS e USF, o item referente às atividades de educação em saúde que viabilizam a identificação de problemas pela população de forma que cada indivíduo reconheça sua co-responsabilidade na preservação de ambientes, bem como, as atividades de promoção do conhecimento das responsabilidades dos órgãos públicos, foi significativamente $(\mathrm{p}=0,044)$ melhor avaliado do que a alternativa concernente às atividades integrativas com os outros serviços que atuam diretamente com os diferentes problemas ambientais identificados (Tabela 3).

$\mathrm{Na}$ análise ajustada para o local de trabalho dos médicos de UBS ou de USF, não foi encontrada diferença estatisticamente significativa $(p=0,238)$ entre os dois grupos.

Quanto ao total de 55 trabalhadores, não foi encontrada diferença significativa $(p=0,166)$ entre as médias dos quesitos relativos às estratégias utilizadas para atuar nos problemas ambientais identificados, na comparação com o local de atuação (UBS ou USF).

\section{DISCUSSÃO}

No conjunto das características-componentes, necessário ao foco socioambiental da APA, tem-se a ação construtiva e integrativa (estratégias) da relação entre saúde e ambiente, para a qual ficou evidente que o conjunto geral dos 55 trabalhadores se identifica mais e, portanto, avalia melhor como estratégia utilizada no nível da atenção básica à saúde o "conhecer os riscos para poder agir/aplicar”, ou seja, o grupo de enfermeiros e de médicos atribuiu maior nota ao quesito referente ao conhecimento das situações ambientais do município, que impliquem em riscos efetivos ou potenciais à saúde humana. Com esta avaliação pode-se dizer que existe relação contextual na característica da ação diagnóstica no enfoque de risco ser predominante no saber dos profissionais de saúde e, portanto, esta ser condição para a ação de programar no processo de trabalho..$^{8-10}$

Acrescenta-se a isso, que as estratégias utilizadas na relação saúde e ambiente, as quais incluíam níveis de participação na gestão integrativa local, no sentido do desenvolvimento de ações em nível 
de formulação de políticas públicas, na participação de projetos, na avaliação e elaboração de instrumentos diagnósticos socioambientais, obtiveram médias bem inferiores.

Esses resultados permitem sugerir que a relação entre a saúde e o ambiente, ${ }^{11}$ no trabalho da atenção básica à saúde, representa um desafio para a organização do trabalho, com vista à identificação, planejamento e intervenção coletiva dos problemas de saúde e ambiente nas comunidades encampadas com o trabalho desenvolvido. Identificar e intervir na relação implicam em saber ambiental, ${ }^{3}$ no qual o conceito de ambiente é "um objeto complexo, integrado por processos de ordem natural, técnica e social, cujas causas e objetivos não podem ser absorvidos num modelo global, por complexo, aberto e holístico que pretenda ser" 3:147

Relacionar saúde e ambiente em seu sentido socioambiental infere de imediato problematizar o sentido de ambiente em sua dimensão física e social como fenômeno desenvolvido nas comunidades e seus municípios.

Não se verificou diferença, entre os 55 trabalhadores (enfermeiros e médicos das USF e UBS), na forma de identificar os problemas ambientais que influenciam a saúde das comunidades, ou seja, esses profissionais agruparam os problemas ambientais em dois conjuntos. O primeiro incluindo os quesitos que melhor foram avaliados como: falta de saneamento básico; presença de vetores de doenças; manejo inadequado de resíduos sólidos e domésticos e ruas sem pavimentação. O segundo como o conjunto dos quesitos com avaliação mais baixa composta por: utilização de agrotóxicos em plantações; alimentos contaminados por agrotóxicos ou outras substâncias; uso indevido dos solos; e erosão e desmatamento.

A relação entre saúde e ambiente, ao longo da história da saúde pública brasileira, esteve aderida a preocupação com o saneamento básico, ${ }^{12} \mathrm{com}$ a área dos resíduos sólidos, industriais e domésticos, com o saneamento rural e com os ambientes de trabalho e a saúde dos trabalhadores, ${ }^{13}$ deixando para outras setores a preocupação com aspectos geofísicos (solos, desmatamento e erosão, por exemplo). Da mesma forma que a preocupação com o ambiente como elemento de interação com o aparecimento de doenças, como fonte de agentes e vetores ligados à cadeia natural de doenças infecciosas, sempre fortemente presente em seu foco de redução ao ambiente como um dos elementos da tríade ecológica. ${ }^{13}$
Este achado remete aos conjuntos de relações contextuais, tradicionalmente construída na sociedade, da formação geral dos profissionais da área da saúde, neste estudo com enfermeiros e médicos, na qual os conteúdos aprendidos pouco conduzem ao campo teórico e metodológico da aplicabilidade de conhecimentos na relação saúde e ambiente, mesmo em nível da atenção básica à saúde e, portanto, do sentido ampliado da saúde, como conceito construído coletivamente ${ }^{6}$ no potencial da relação com o ambiente.

Importa afirmar ser necessário no campo da saúde, a aproximação enquanto conceito e prática das categorias saúde e ambiente, valorizando assim o ambiente como determinante/condicionante no processo saúde-doença humanos, assim como na área ambiental, a visualização das alterações ambientais na saúde humana, contribuiria para ampliar a preocupação com o impacto na vida humana do enfoque ambiental, superando a valorização dos impactos ambientais somente sobre o meio físico. ${ }^{12}$

A existência de problemas ambientais globais, ${ }^{14}$ é reconhecida em diferentes áreas, grupos sociais e de diferentes formas, os quais se encontram relacionados com as mudanças climáticas, a diminuição da camada de ozônio, como exemplo; associado a este cenário geral e abrangente entende-se que os municípios e os bairros, participam em maior ou menor escala da influência nessas mudanças globais e nesses locais, inclui-se a vida cotidiana de cada indivíduo e de cada grupo social, passando assim, de problema ou problemática socioambiental global para particular e local. ${ }^{14}$ Entende-se que a maioria dos problemas ambientais são de caráter local e repercutem diretamente na saúde e qualidade de vida dos seres humanos. ${ }^{1}$

Portanto, torna-se imprescindível o reconhecimento desses problemas de natureza local e potencial global para a aderência da estratégia da APA, no foco socioambiental do trabalho na atenção básica à saúde. Visto que esta estratégia possibilita organizar o trabalho de maneira a assumir o ambiente (em sentido social e natural) como constituinte do objeto a ser transformado em produto do processo de trabalho.

Para atuar nesse "objeto”, ou seja, na relação saúde e ambiente, com enfoque socioambiental, no conjunto de ações participativas e integrativas com as comunidades, têm-se como instrumentos processuais importantes na APA, as ações de educação em saúde, ${ }^{14}$ como aquelas cujas médias atingiram escores 
melhores, tanto para os enfermeiros como para os médicos, mas somente ocorreu diferença significativa entre as médias das avaliações na comparação com o outro quesito (atividades integrativas com os outros serviços que atuam diretamente com os diferentes problemas ambientais) no grupo geral dos médicos. Esses achados expressam a diferença na identificação dos médicos respondentes, no trabalho neste nível de assistência, para a utilização de estratégias junto às comunidades.

O resultado desta análise possibilita afirmar que o conjunto dos enfermeiros das USF e UBS, sujeitos deste estudo, não difere na avaliação o instrumento processual da educação em saúde, bem como das ações gerenciais que integram as atividades com os outros setores que atuam diretamente nos problemas ambientais identificados nas comunidades, no conjunto do sistema local, como integrantes de seu processo de trabalho na atenção básica à saúde. No entanto, as atividades de natureza integrativa com outros setores, que conduzem as ações sobre o meio ambiente no foco da saúde, em seus diferentes cenários da organização local do sistema de saúde ampliado (vigilância à saúde, secretaria do meio ambiente, serviços urbanos entre outros, ligados aos órgãos públicos, como exemplos), receberam as menores notas em ambas categorias profissionais - enfermeiros e médicos.

Ao finalizar a discussão da análise dos dados, considerando o conjunto dos 55 trabalhadores (enfermeiros e médicos), conduz a referência de que a estrutura organizativa da atenção básica à saúde em equipes de saúde da família e em equipes tradicionais, ainda não influencia significativamente a utilização de estratégias no trabalho para atuar na relação saúde e ambiente; na identificação de problemas ambientais que influenciam na saúde das comunidades e; nas formas para atuar junto às comunidades nos problemas ambientais identificados. Além disso, foi possível verificar que o item referente ao conhecimento das situações ambientais do município que impliquem em riscos efetivos ou potenciais à saúde humana é a estratégia melhor identificada pelas duas categorias para trabalhar a relação entre saúde e ambiente.

\section{CONSIDERAÇÕES FINAIS}

Ao ser apreendido o enfoque socioambiental do trabalho, por meio do conjunto geral dos trabalhadores - os enfermeiros e os médicos - na atenção básica à saúde, fica explícito a necessidade de se intensificar os instrumentos processuais para a construção do reconhecimento e decisão sobre os problemas e problemáticas socioambientais das comunidades locais de inclusão no processo de trabalho. Neste sentido, o desenvolvimento de políticas saudáveis e sua aderência ao trabalho diário dos profissionais, é de relevância vital para a concretude do enfoque socioambiental da APA - na relação saúde e ambiente.

Para que a $\mathrm{APA}^{1-2}$ seja desenvolvida num contexto socioambiental do trabalho, ${ }^{6}$ torna-se necessário a construção de estratégias que relacionem saúde e ambiente, ou seja, é preciso que os trabalhadores desenvolvam estratégias para a ação construtiva e integrativa da relação entre saúde e ambiente, ${ }^{8}$ no trabalho da atenção básica à saúde. Para que isto ocorra faz-se imperativo que esta relação esteja no "objeto de trabalho" a ser transformado em produto, por meio de estratégias coletivas e sustentáveis. Além dessa inclusão, a relação saúde-ambiente deve existir nas próprias necessidades sociais que serão assumidas como finalidades no trabalho em saúde. Pode-se reiterar que estratégias sustentáveis compreendem "um processo que permite satisfazer as necessidades da população atual sem comprometer a capacidade de atender as gerações futuras". 3:19

Nesta direção, o presente estudo encontra nesta característica o seu próprio limite, ou seja, a apreensão de um objeto complexo e ao mesmo tempo, provocador de impasses e embates ideológicos, que permitam ou não, as possibilidades da estratégia socioambiental da APA no trabalho da atenção básica à saúde.

$\mathrm{Na}$ interdisciplinaridade do trabalho em saúde, e na enfermagem em particular, por seu potencial histórico de ação a valores básicos $\mathrm{da}$ atenção à saúde, pode ser estratégica para assegurar a viabilidade e o alcance de mudanças importantes, tanto em nível da consciência comunitária, ${ }^{8}$ como de políticas do Estado, que disseminem fundamentos e estruturem a atenção primária ambiental.

O pressuposto de que a APA no trabalho da atenção básica à saúde, na relação entre saúde e as questões ambientais têm potencial "qualificador" para a ação pública e integrativa na solução dos problemas e problemáticas locais, no foco da saúde socioambiental, foi o desencadeador deste estudo, o qual em sua formalidade textual é finalizado e, no mesmo tempo histórico reiniciado, em seu conteúdo complexo e empolgante. 


\section{REFERÊNCIAS}

1 Organização Mundial da Saúde, Organização PanAmericana da Saúde. Atenção Primária Ambiental. Brasília (DF): Gráfica e Ed. Brasil; 2000.

2 Leff E. Epistemiologia ambiental. São Paulo (SP): Cortez; 2000.

3 Leff H. Saber Ambiental: sustentabilidade, racionalidade, complexidade e poder. Petrópolis (RJ): Vozes; 2001.

4 Minayo MCS. Enfoque ecossistêmico de saúde e qualidade de vida. In: Minayo MCS, Miranda AC, organizadores. Saúde e ambiente sustentável: estreitando nós. Rio de Janeiro (RJ): Ed. FIOCRUZ/ ABRASCO; 2002. p.173-90. 5 Loureiro CFB, Azaziel M, Franca N. Educação Ambiental e gestão participativa em unidades de conservação. Rio de Janeiro (RJ): IBASE/IBAMA; 2003.

6 Cezar-Vaz MR, Soares MCF, Martins SR, Sena J, Santos LR, Rubira LT, et al. Saber ambiental: instrumento interdisciplinar para a produção de saúde. Texto Contexto Enferm. 2005 Jan-Abr; 14 (4): 391-7.

7 Marx K. O capital: crítica da economia política. 10a ed. Liv.I v.1 São Paulo (SP): Difel; 1985.

8 Cezar-Vaz MR, Cabreira GO, Couto ZFS, Soares JFS, Weis A, Berto J. O trabalho da enfermeira na atenção básica de saúde: assumindo uma forma programática para o conteúdo clínico-social. Texto Contexto Enferm. 2003 Ago-Out; 12 (3): 342-50.

9 Almeida MCP, Mishima SM, Silva EM, Mello DF. $O$ trabalho de enfermagem e sua articulação com o processo de trabalho em saúde coletiva na Rede Básica de Saúde. In: Almeida MCP, Rocha SMM, organizadores. O trabalho da enfermagem. São Paulo (SP): Cortez; 1997. p.61-112.

10 Ribeiro EM, Pires D, Blank VLG. A teorização sobre processo de trabalho em saúde como instrumental para a análise do trabalho no Programa Saúde da Família. Cader. Saúde Pública 2004 Mar-Abr; 20 (2): 438-46.

11 Leff E, organizador. A complexidade ambiental. São Paulo (SP): Cortez; 2003.

12 Heller L. Relação entre saúde e saneamento na perspectiva do desenvolvimento. Ciência Saúde Coletiva. 1998 Abr-Jun; 3 (2): 73-84.

13 Tambellini AT, Câmara VM. A temática saúde e ambiente no processo de desenvolvimento do campo da saúde coletiva: aspectos históricos, conceituais e metodológicos. Ciência Saúde Coletiva. 1998 AbrJun; 3 (2): 47-59.

14 Hannigan JA. Sociologia ambiental: a formação de uma perspectiva social. Lisboa (PT): Instituto Piaget/ Stória; 1995. 